\title{
Determination of Aortic Distensibility Using Non-rigid Registration of Cine MR Images
}

\author{
Maria Lorenzo-Valdés ${ }^{1}$, Gerardo I. Sanchez-Ortiz ${ }^{1}$, Hugo Bogren ${ }^{2 \star}$, \\ Raad Mohiaddin ${ }^{2}$, and Daniel Rueckert ${ }^{1}$ \\ 1 Visual Information Processing Group, Department of Computing, \\ Imperial College London, 180 Queen's Gate, London SW7 2BZ, United Kingdom \\ 2 Royal Brompton and Harefield NHS Trust, Sydney Street, \\ London, United Kingdom
}

\begin{abstract}
A novel method for the estimation of areas in 2D MR images of the aorta is presented. The method uses spatio-temporal non-rigid registration in order to obtain the $2 \mathrm{D}$ deformation fields of the vessels during the cardiac cycle. This is accomplished by aligning all time frames in the image sequence simultaneously to the first one. The determinant of the Jacobian of the 2D deformation fields are then computed to obtain the expansion (or contraction) at each time frame, with respect to the first time frame. By using 3D splines, the method exploits the relation between time frames in order to obtain continuous and smooth distensibility measurements throughout the cardiac cycle. Validation was carried out with MR images of the aorta. Experiments for the registration and estimation of areas in the aorta are presented in 60 data sets corresponding to three different sections of the aorta (proximal, mid and distal) in 20 different subjects, where each set consisted of 17 to 38 time frames. Manually estimated areas are compared to the areas estimated automatically in 8 data sets where the average error is $2.3 \%$ of the area manually obtained.
\end{abstract}

\section{Introduction}

The assessment of the elasticity of the great vessels is important in order to evaluate cardiovascular disease and performance since it reflects aging and atherosclerosis 12 . In addition, it can also be used to evaluate the results of plaquereducing therapies. This elasticity assessment is usually accomplished by measuring compliance or distensibility, which are wall properties of great vessels. Arterial compliance reflects the buffering capacity of an artery and is defined as the absolute change in volume or cross-sectional area per unit of pulse pressure $(\triangle V / \triangle P)$. Decrease in compliance increases cardiac after-load and the risk of cardiac hypertrophy. Arterial distensibility is defined as the relative change in volume or cross-sectional area per unit of pulse pressure $([\triangle V / V] / \triangle P)$ and reflects mainly the elasticity of the wall.

\footnotetext{
* Hugo Bogren on sabbatical leave from the Department of Radiology, University of California Davis School of Medicine, California, USA
} 
Two approaches for measuring compliance and distensibility are normally used. The first approach uses instantaneous pressure-dimension relations of single pulse waves at varying levels of distending pressures. A pulse wave travels with a speed of several metres/seconds from the aorta to the arterial branches. This velocity can be measured by recording the pressure pulse waves at two places with Doppler echo-probes [3], or using phase contrast MR 112. The pulse wave velocity along the vessel of interest provides a measure of compliance $C$ through the approximate relation $C=1 /\left(c^{2} \rho\right)$, where $c$ is the pulse wave velocity and $\rho$ is the blood mass density which can be considered to be constant. The second approach for obtaining compliance and distensibility uses measurements of area and pressure changes. While measuring pressure is simple to carry out for the recordings at end-systole and end-diastole using a sphygmomanometer, the measurement of volume changes is more difficult because there is no simple means of estimating regional changes in blood volume of a vessel. However, assuming that there is mainly radial and negligible axial vessel movement during pulse pressure, compliance and distensibility can be estimated as a change in radius, diameter, or cross-sectional area for a given change in pressure.

Manual delineation of the great vessels is usually performed in order to compute the area, however it is time consuming and there is a significant intra and inter-observer variability which make the results inconsistent. Deformable models have been previously proposed for the tracking and segmentation of the aorta [4. However, this approach has not been applied on continuous measurements through a complete cardiac cycle.

This paper proposes a method to estimate the area changes of the aorta from MR images. We have successfully applied a similar approach for tracking cardiac MR image sequences [5]. First, each time frame of the image sequence is registered to the first frame using non-rigid registration to obtain the deformation fields of the aorta during the cardiac cycle. Subsequently, to calculate regional area changes the determinant of the Jacobian of the deformation field is integrated over the area of interest to quantify differences between registered images and the reference frame.

\section{2D Registration of Cine MR Images}

The purpose of image registration is to find the optimal correspondence between anatomical regions in two different images. Registration is a technique where the geometrical relation between two images is described by a transformation that maps points in one image to its corresponding point in another image [6]. Registration can be accomplished using different types of transformations that can be classified as rigid or non-rigid.

In our application non-rigid registrations are performed between a baseline (or reference) image at time $t=0$ and all other images in the sequence to obtain transformations that can describe the deforming soft tissue such as the wall of the great vessels.

To model local deformations Rueckert et al. [7] proposed a non-rigid transformation algorithm based on free-form deformations and B-splines. It manip- 
ulates control points allowing deformation of the shape of the $2 \mathrm{D}$ or $3 \mathrm{D}$ object producing a smooth and continuous transformation:

$$
T_{\text {local }}(x, y)=\sum_{m=0} \sum_{n=0} B_{m}(u) B_{n}(v) \phi_{i+m, j+n}
$$

where $i=\left\lfloor x / n_{x}\right]-1, j=\left\lfloor y / n_{y}\right]-1, u=x / n_{x}-\left\lfloor x / n_{x}\right\rfloor, v=y / n_{y}-\left\lfloor y / n_{y}\right\rfloor(\lfloor$ ] means round down) and where $B_{m}$ represents the $m$-th basis function of the B-spline described in [8].

To relate both images, a measurement of alignment is needed to find the correct transformation. If the images to be registered belong to the same image sequence, we can assume that the intensities in images $A$ and $B$ are closely related and Viola [9] demonstrated that in this case the correlation coefficient (CC) may be considered as the ideal similarity measure. For images $A$ and $B$, the aim of the registration process is to find the transformation $T$ which maximises:

$$
C C=\frac{\sum_{i}\left(A\left(x_{i}\right)-\bar{A}\right)\left(B\left(T\left(x_{i}\right)\right)-\bar{B}(T)\right)}{\left\{\sum_{i}\left(A\left(x_{i}\right)-\bar{A}\right)^{2} \sum_{i}\left(B\left(T\left(x_{i}\right)\right)-\bar{B}(T)\right)^{2}\right\}^{1 / 2}} \quad \forall i \in A \cap B
$$

where $\bar{A}$ and $\bar{B}$ are the mean intensity values of voxels in image $A$ and the transformed image $B$, respectively.

The resulting transformation maps each point of the image reference $A$ to the corresponding point in the image $B$. After the optimal transformation has been obtained, the resulting deformation field can be analysed to identify areas which have expanded or contracted. This analysis requires the calculation of the determinant of the Jacobian matrix of the deformation field which defines the local volume change in the neighbourhood of a point $\mathbf{x}$.

\section{3D Registration of Cine MR Images and Estimation of Area Changes in the Aorta}

The idea of the proposed approach is to estimate the expansion or contraction of the aorta in different time frames with respect to a baseline image of the vessel. While the registration technique described in the previous section can be used to register all time frames in a sequence to their respective baseline image, it does not exploit the temporal coherence of the image sequence. In this section we propose a modified spatio-temporal registration which exploits the coherence of the image sequence.

Rather than considering each 2D cross-sectional image separately we use the $2 \mathrm{D}$ image sequence as a $3 \mathrm{D}(2 \mathrm{D}+$ time $)$ image, where $x, y$ describe the crosssectional position and $t$ the time during cardiac cycle. The goal of the spatiotemporal registration is to find the optimal transformation $T:(x, y, t) \mapsto(x, y, 0)$ which maps all points in the $3 \mathrm{D}$ image $I_{\text {sequence }}(x, y, t)$ to their corresponding anatomical location in the $2 \mathrm{D}$ baseline image $I_{\text {baseline }}(x, y)$.

Next, the image sequence $I_{\text {sequence }}$ is registered to the baseline image $I_{\text {baseline }}$ to find the optimal transformation $T$ as presented in Fig 1 For this purpose we 


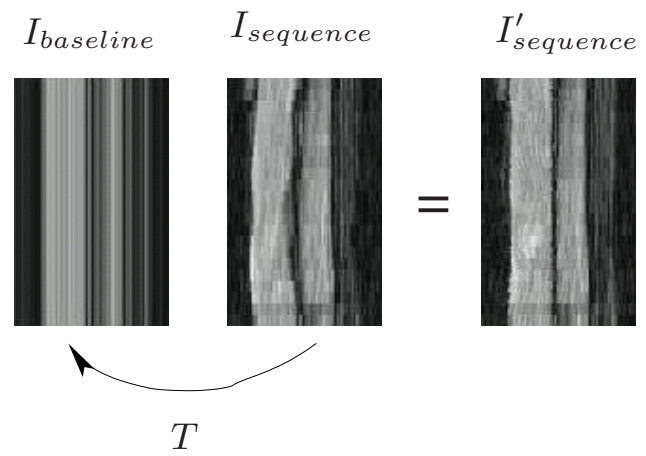

Fig. 1. Registration of the image sequence $I_{\text {sequence }}$ with the baseline image $I_{\text {baseline }}$.

modify eq. (1) to ensure a temporally smooth transformation:

$$
\mathbf{T}_{I_{0} I_{N}}(x, y, t)=\sum_{t=0}^{3} \sum_{m=0}^{3} \sum_{n=0}^{3} B_{l}(u) B_{m}(v) B_{n}(w) \phi_{i+l, j+m, k+n}
$$

Note that this transformation is a mapping from $3 \mathrm{D}(x, y, t)$ to $2 \mathrm{D}(x, y, 0)$, i.e. the displacement of the control points is restricted to the $x$ and $y$ directions only. By optimising the location of all control points simultaneously we are able to simultaneously register all time frames of the image sequence to the baseline image. As a result we ensure a smooth deformation between time frames.

To calculate the change in area of a structure of interest we need to integrate the determinant of the Jacobian over the area of interest $\Omega$ in the baseline image that is on the region of the aorta. At any given point $(x, y, t)$ the determinant of the Jacobian can be computed as

$$
J(x, y, t)=\left[\begin{array}{ll}
\frac{\partial u_{x}}{\partial x} & \frac{\partial u_{x}}{\partial y} \\
\frac{\partial u_{y}}{\partial x} & \frac{\partial u_{y}}{\partial y}
\end{array}\right]
$$

where $\frac{\partial u_{i}}{\partial j}$ is the rate of change of the $i_{t h}$ component of the displacement field $u$ in the direction $j$. Note that this Jacobian only takes the spatial component of the transformation into account. The determinant of the Jacobian quantifies the expansion or contraction between the image sequence and the baseline image, for any given location in the baseline coordinate system. The resulting Jacobian values for frame $t$ contained in the selected region $\Omega$ were averaged for each time frame.

$$
\bar{J}_{n}=\frac{\int_{\Omega} J_{x y} d x d y}{\int_{\Omega} d x d y}=\frac{\sum_{k} J_{x y}(k)}{K} \forall k \in T_{I(0) I(n)}(x, y) \cap \Omega
$$

where $\bar{J}_{n}$ is the average (or global) ratio of expansion or contraction of frame $n, J_{x y}(k)$ is the Jacobian of any transformed point found in the region $\Omega$, and $K$ is the total number of transformed points (or image voxels) in the region $\Omega$. This would describe the global expansion or contraction ratio of the aorta at 
that particular time frame. The segmented area in the first time frame is then multiplied by this ratio to obtain the area in each time frame. In this way, not only the actual expansion ratio values but the absolute area changes of the vessel throughout a complete cardiac cycle can be visualised and used to estimate the elasticity of the vessel.

\section{Experiments and Results}

Images were acquired at Royal Brompton Hospital, London, UK, using a Siemens Sonata $1.5 \mathrm{~T}$ scanner with a TrueFisp sequence. Images of the proximal, mid and distal parts of the ascending aorta were obtained from 20 different subjects with a resolution of $192 \times 256$ and pixel size around $0.6 \times 0.6 \mathrm{~mm}$ with a slice thickness of $6 \mathrm{~mm}$. The images were taken perpendicular to the aorta. For each subject between 17 and 38 time frames were acquired, at intervals of approximately $25-50 \mathrm{msec}$. We divided the subjects in two age groups. The age of 10 subjects ranged between 23 and 34 years old and the age of the other 10 subjects ranged between 57 and 80 years old.

The proposed approach was applied to the 60 data sets from 20 subjects. First, the area change with respect to the first time frame image was computed with the proposed method. In five data sets the second or third time frame images were taken as the reference instead of the first time frame image because in those cases the first time frame image did not have good contrast and the boundaries of the aorta were not well defined. Therefore, frames with a better quality were chosen as the reference. Fig. 2] shows the expansion/contraction ratio of the cross-sectional area of the aorta in the three different sections (proximal, mid and distal) calculated with respect to the area of the first time frame. It presents the results for all the data sets of the younger and older subjects. The expansion of most of the older subjects do not exceed $120 \%$ of the area estimated in the first frame.

In order to evaluate our method, manual segmentations were performed by an expert observer in the complete time sequences of eight image sets where double determinations (i.e. two manual delineations) were averaged. The manually estimated area of the vessel was compared to those obtained with the proposed method. Fig. 3 presents the area manually estimated by the expert and by the proposed approach. Table 1 summarises the maximum difference between the manual and automatic estimates of the area. The maximum difference is $5.8 \%$ on average, while the average difference over all is $2.3 \%$. For volunteer 6 the maximum difference between both areas is $9.9 \%$, however, the source of this error is due to an inconsistent manual segmentation of the second time frame as can be seen in Fig. 3 .

In order to visualise the results of the complete time sequence, using the transformation computed by the registration algorithm we have transformed the segmented image of the first time frame into the shape corresponding to the remaining time frames. This allows us to see the projected segmentation (or $2 \mathrm{D}$ delineation) of the vessels throughout the cardiac cycle. Figure 4 shows an 

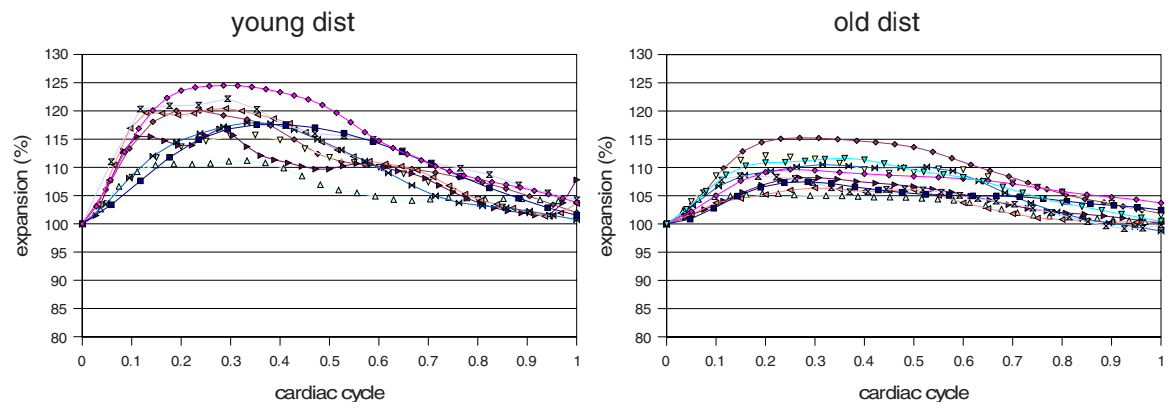

young mid

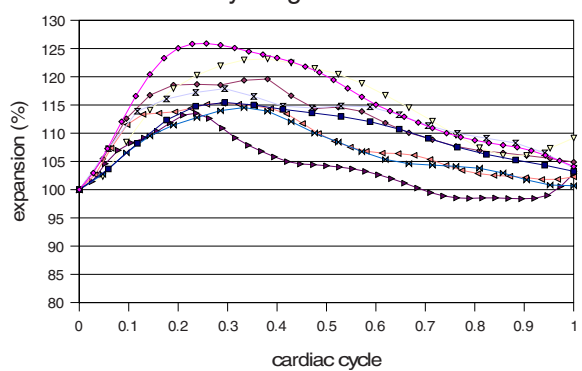

old mid

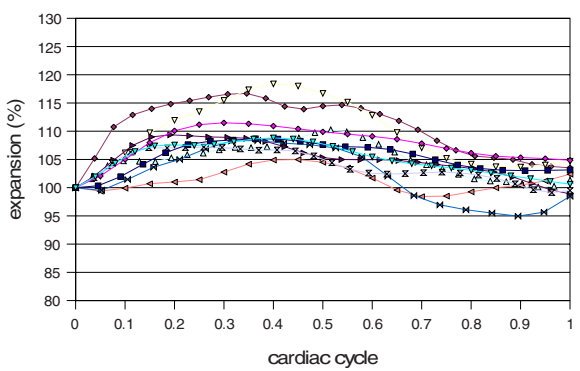

young prox

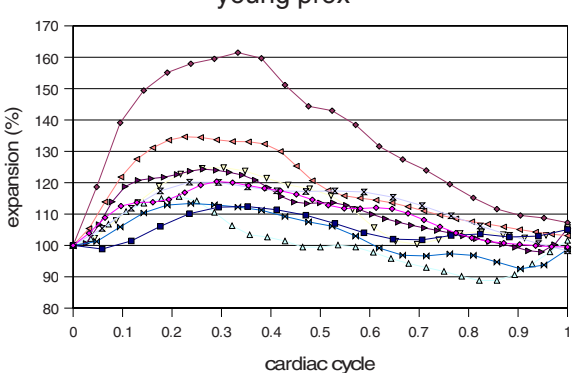

old prox

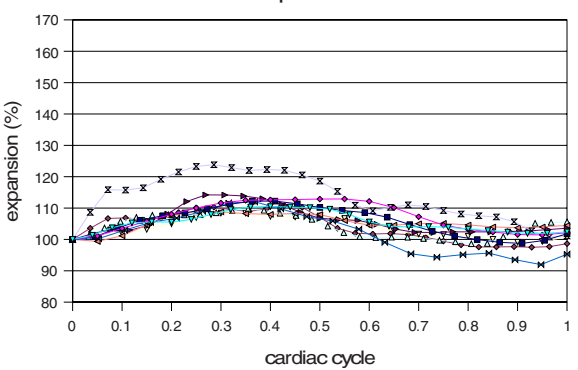

Fig. 2. Automatic estimation of the expansion/contraction ratio of the aorta with respect to the first time frame.
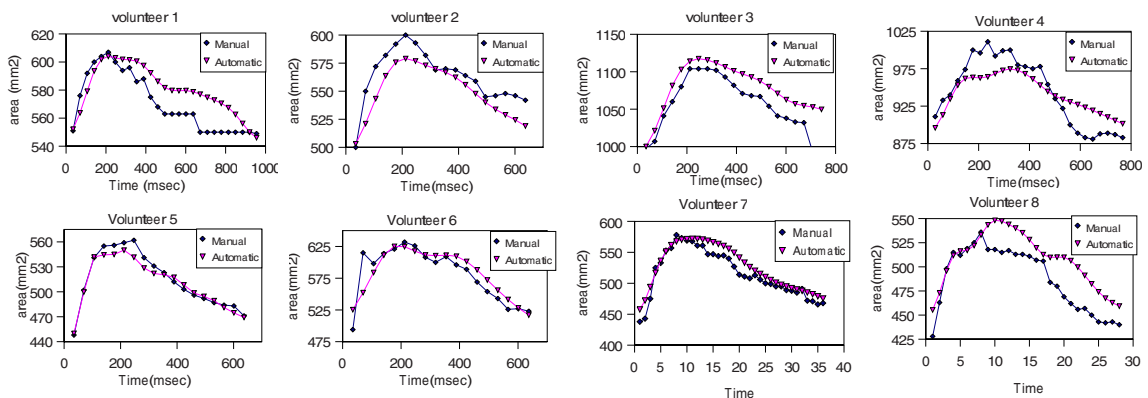

Fig. 3. Example of the manual and automatic estimation of the areas in a set of images. Notice the smooth time variations for the proposed method. 
example of segmentation results on a cross-sectional image. Figure 5 illustrates the segmentation results on a $3 \mathrm{D}(2 \mathrm{D}+$ Time) representation.

Table 1. Differences between the area estimated manually and automatically for each curve of Fig. 3 divided by the area manually estimated.

\begin{tabular}{|c|c|c|c|c|c|c|c|c|c|}
\hline Subject & 1 & 2 & 3 & 4 & 5 & 6 & 7 & 8 & Average \\
\hline Maximum difference & $4.4 \%$ & $4.8 \%$ & $5.5 \%$ & $4.6 \%$ & $3.6 \%$ & $9.9 \%$ & $5.1 \%$ & $8.7 \%$ & $5.8 \%$ \\
\hline Average difference & $2.0 \%$ & $2.3 \%$ & $2.0 \%$ & $2.5 \%$ & $1.1 \%$ & $2.2 \%$ & $2.0 \%$ & $4.1 \%$ & $2.3 \%$ \\
\hline
\end{tabular}
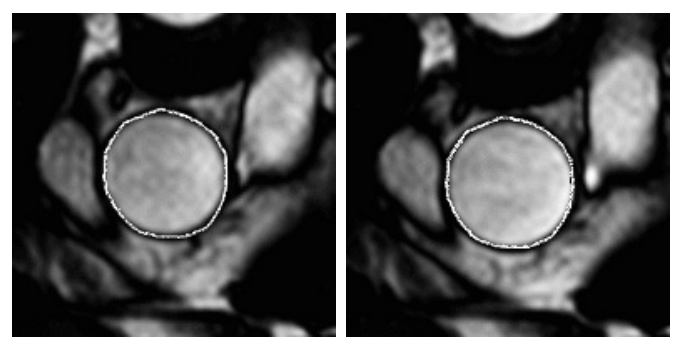

Fig. 4. Example of segmentation results shown on a cross section at (a) end-diastole and (b) end-systole. In order to help visualising results in this non-colour print, the continuous segmentation contour is shown as a dotted line.

\section{Discussion}

We have presented a procedure to estimate the transverse area in MR images of the aorta. The registration yields a deformation field from which the expansion or contraction ratio of the aorta could be accurately estimated. The 8 examples in Fig. 3 show that the area-time curve estimated by the proposed approach is smooth while the manual estimate of the area-time curve is noisy and has some abrupt changes due to the different criteria of the expert to segment the aorta in different time frames. The manual method was very time consuming with $60-100$ measurements per section and took approximately one hour while the automatic method took 5 minutes once the program was set up. The automatic contours were consistent with no variation, contrary to the manual double determinations, and appeared more reliable. The method relates the images in time and the results are promising for physiological and clinical studies in the assessment of elasticity of the aorta as shown in Fig. 2 where the difference in elasticity between young and old subjects can be observed. The method is limited when the first time frame does not have a good contrast, however another time frame with better contrast can be chosen as a reference and the expansion/contraction ratio is calculated for that reference. At present only one time frame per sequence needs to be segmented manually. Future work will involve the automatic 


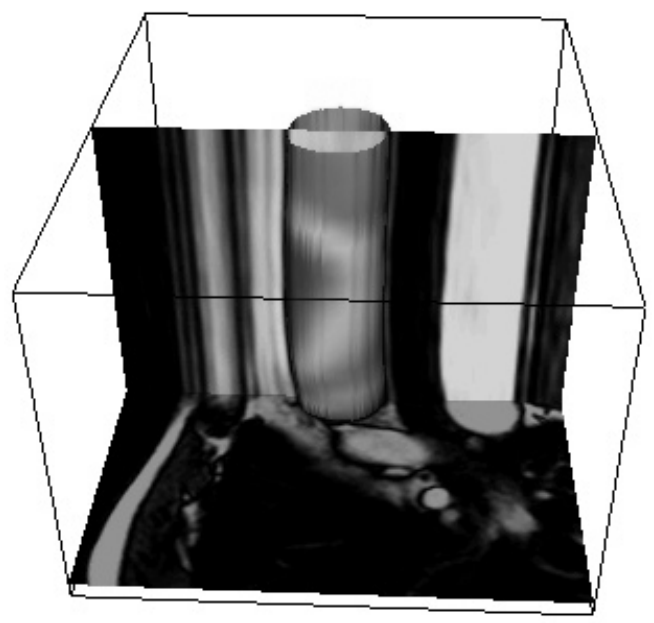

Fig. 5. Example of automatic segmentation results shown on a $3 \mathrm{D}(2 \mathrm{D}+$ Time $)$ representation. The vertical axis $(t)$ shows the time evolution of a section of the aorta. The colours on the segmentation surface show the magnitude of deformation in every region and at every time (blue is the minimum and red the maximum value).

segmentation of the first frame in order to make the approach fully automatic and measurements in the ascending and descending aorta, the aortic arch and the main, left and right pulmonary arteries will be performed.

\section{References}

1. R. H. Mohiaddin, S. R. Underwood, H. G. Bogren, D. N. Firmin, R. H. Klipstein, R. S. Rees, and D.B. Longmore. Regional aortic compliance studied by magnetic resonance imaging: The effects of age, training and coronary artery disease. British Heart Journal, 62:90-96, 1989.

2. H. G. Bogren, R. H. Mohiaddin, R. K. Klipstein, D. N. Firmin, R. S. Underwood, S. R. Rees, and D. B. Longmore. The function of the aorta in ischemic heart disease: a magnetic resonance and angiographic study of aortic compliance and blood flow patterns. Am. Heart J., 118(2):234-247, 1989.

3. J. S. Wright, J. K. Cruickshank, S. Kontis, C. Dore, and R. G. Gosling. Aortic compliance measured by non-invasive doppler ultrasound: description of a method and its reproducibility. Clinical Science, 78:463-468, 1990.

4. D. Rueckert, P. Burger, S. M. Forbat, R. D. Mohiaddin, and G. Z. Yang. Automatic tracking of the aorta in cardiovascular MR images using deformable models. IEEE Transactions on Medical Imaging, 16(5):581-590, 1997.

5. M. Lorenzo-Valdes, G. I. Sanchez-Ortiz, R. Mohiaddin, and D. Rueckert. Atlasbased segmentation and tracking of 3D cardiac MR images using non-rigid registration. In Fifth Int. Conf. on Medical Image Computing and Computer-Assisted Intervention (MICCAI 'O2), Lecture Notes in Computer Science, pages 642-650. Springer-Verlag, 2002. 
6. J. M. Fitzpatrick, D. L. G. Hill, and C. R. Maurer, Jr. Image registration. In Milan Sonka and J. Michael Fitzpatrick, editors, Handbook of Medical Imaging, volume 2, pages 447-513. SPIE Press, 2000.

7. D. Rueckert, L.I. Sonoda, C. Hayes, D.L.G. Hill, M.O. Leach, and D.J. Hawkes. Nonrigid registration using free-form deformations: Application to breast MR images. IEEE Transactions on Medical Imaging, 18(8):712-721, 1999.

8. S. Lee, G. Wolberg, and S. Yong Shin. Scattered data interpolation with multilevel B-splines. IEEE Transactions on Visualization and Computer Graphics, 3(3):228244, 1997.

9. P. Viola. Alignment by Maximization of Mutual Information. PhD thesis, Massachusetts Institute of Technology, 1995. 